\title{
Linx
}

Revue des linguistes de l'université Paris X Nanterre

5 | 1994

La négation

\section{La négation et les théories de l'illocutoire}

\section{Bruno Callebaut}

\section{(2) OpenEdition \\ Journals}

Édition électronique

URL : http://journals.openedition.org/linx/1208

DOI : 10.4000/linx.1208

ISSN : 2118-9692

\section{Éditeur}

Presses universitaires de Paris Nanterre

\section{Édition imprimée}

Date de publication : 1 juin 1994

Pagination : 191-203

ISSN : 0246-8743

\section{Référence électronique}

Bruno Callebaut, «La négation et les théories de l'illocutoire », Linx [En ligne], 5 | 1994, mis en ligne le

18 juillet 2012, consulté le 19 avril 2019. URL : http://journals.openedition.org/linx/1208 ; DOI :

10.4000/linx.1208

Ce document a été généré automatiquement le 19 avril 2019.

Département de Sciences du langage, Université Paris Ouest 


\title{
La négation et les théories de l'illocutoire
}

\author{
Bruno Callebaut
}

\section{Introduction}

1 Dans ma thèse (Callebaut 1991) j'ai étudié la négation à la lumière des théories pragmatiques, en l'examinant comme marqueur potentiel d'actes de langage spécifiques. Pour ce colloque, tout en restant dans la même perspective pragmatique, j'adopte l'attitude inverse, en étudiant les actes de langage à la lumière des effets que la négation a sur eux. Même si de la sorte les projecteurs seront moins braqués sur la négation que sur les théories illocutoires, c'est toujours en lui rendant un hommage mérité; il apparaitra en effet, $\mathrm{j}$ 'espère, combien la négation peut rester au coeur de toute heuristique linguistique.

2 Je propose ici deux pistes de recherche: d'abord une réflexion plus théorique sur la classification des actes de langage en rapport avec la négation (1), ensuite une application concrète à propos des effets de la négation sur les énoncés contenant un verbe à «potentiel illocutoire» $(2)$.

\section{Les actes de langage et la négation}

\section{1 .}

Comme il existe déjà une vaste littérature, je me limite ici à rappeler que tout a commencé avec la distinction entre énoncés performatifs et constatifs, due essentiellement à Austin. On en donne souvent les exemples contrastés «La séance est levée » (prononcé par le président de l'assemblée, - performatif atypique, parce que à la troisième personne) et «La séance est levée dans la plus grande confusion » (énoncé constatif émanant du journaliste qui assiste en observateur). Renvoyons le lecteur intéressé par le performatif 
aux nombreuses études de Récanati, mais aussi aux prises de position stimulantes de Berrendonner (e.a. 1981) et à l'excellente thèse de F. Nicoloff (1984).

On le sait, les critiques à l'encontre de cette distinction ont amené les spécialistes (Austin lui-même et surtout ensuite Searle) à élaborer une théorie plus large des valeurs pragmatiques attachées aux énoncés : la théorie de l'illocutoire. Tout énoncé peut être analysé dans ses valeurs locutoire, illocutoire et perlocutoire. Le locutoire touchant des aspects plus proprement sémantiques, et le perlocutoire les effets secondaires, d'ordre psychologique, les pragmaticiens se sont tournés surtout vers l'illocutoire: la valeur intentionnelle traduisant les visées de l'énonciateur. Searle a affiné la typologie des actes illocutoires esquissée par Austin, dans un modèle qui est désormais classique et bien connu. Sur base d'un faisceau de douze paramètres, se laissant réduire à quatre types de conditions, il distingue cinq grandes classes d'actes illocutoires : les actes représentatifs, les actes directifs, les actes commissifs, les actes expressifs et les actes déclaratifs. Je reprends ici les termes les plus usuels et m'en tiendrai à une présentation succincte (on en trouve les détails chez tous les pragmaticiens, p. ex. Moeschler 1985). Des critiques de cette typologie se trouvent encore une fois dans Récanati $(1977,1981)$, mais aussi dans la littérature allemande (v. p. ex. Ballmer dans Grewendorf, 1979). Les difficultés réelles qu'on rencontre dans l'application du modèle searlien ne résident d'ailleurs pas forcément dans des défauts de la typologie elle-même.

5 Toute la théorie de l'illocutoire garde de son origine austinienne un héritage déterminant en ceci que les analyses continuent à s'intéresser aux énoncés à la première personne plus qu'aux autres. Comme pour la performativité, la reconnaissance d'une valeur illocutoire est plus pertinente pour ces énoncés où l'énonciateur entre lui-même dans son énonciation. J'ai choisi, pour la deuxième partie aussi, d'étudier des emplois de verbes «à potentiel illocutoire » à la première personne.

6 Nous allons parcourir les cinq classes en évaluant leur comportement face à la négation, dans le but de réévaluer la typologie même et la théorie qui en est à la base. Dans l'analyse interne des actes (concernant les quatre conditions ou critères), nous nous arrêterons surtout à la condition dite " essentielle ", c'est-à-dire au but illocutoire ou en d'autres termes au type d'obligation contractée par le locuteur ou l'interlocuteur (à ce à quoi il s'engage par l'énonciation qui produit l'acte). Mais la question se posera aussi de l'effet de la négation sur une autre condition essentielle, la condition de contenu propositionnel, qui spécifie la nature du contenu de l'acte (assertion, ordre, question, requête...). En revanche, les « conditions préliminaires » et « de sincérité », indiquant les dispositions internes du locuteur, ne se laissent pas examiner dans ce cadre. Nous en sommes toujours réduits à examiner le décodage d'énoncés observables, et à expérimenter sur leur polarité positive ou négative.

7 Un facteur qui joue pour tous les types d'actes est leur caractère "primaire " ou « explicite ». Cela intervenait d'ailleurs déjà dans la distinction originelle entre énoncés performatifs et constatifs. On se rend compte par exemple qu'un acte d'avertissement ne se doit pas d'être introduit par une formule explicite "je t'avertis (qu'il y a un taureau...) ». En fait, cela sera même plutôt exceptionnel et on aura en règle des énoncés plus directs, primaires, comme « (attention) il y a un taureau dans le pré !». L'explicitation d'un acte demande toujours à être interprétée : que se passe-t-il quand en même temps qu'on pose un acte on ressent le besoin de le nommer? La distinction entre actes primaires et explicites s'avèrera importante. 


\subsubsection{Les actes expressifs} et on mentionne comme types l'acte de féliciter, de s'excuser, de remercier, de saluer, de se plaindre etc. Si on l'interprète aussi largement que le permet cette définition floue, c'est une classe hétérogène, à cheval d'ailleurs sur la distinction entre actes performatifs (dans les actes sociaux institutionnalisés) et constatifs (dans l'expression des états psychologiques). On peut se demander où prendraient place ici les expressions de type « zut ! etc., interjections qui renvoient bien à la fonction " expressive » ou " émotive » de la typologie fonctionnelle de Jakobson. Le comportement variable de tous ces actes face à la négation confirme cette hétérogénéité.

9 A. Primaires : les types plus constatifs admettent sans problème la négation; les états psychologiques se laissent exprimer dans les deux polarités positive ou négative :

«Je ne suis pas très content de votre progression »,

"Je ne regrette pas ma visite",

« ce n'est pas très gentil ».

On remarquera que je ne considere pas les exemples précédents (malgré la première personne) comme « explicitant » une valeur illocutoire ; ils restent à mon avis primaires.

11 Les types plus performatifs en revanche, dans leur expression directe, primaire, (« salut », « merci », « mes félicitations » etc.), du moins si on leur garde cet aspect formulaire, ne se laissent pas nier!

B. Explicites : l'acte expressif peut être marqué explicitement par l'enchâssement et/ou l'explicitation du je énonciateur dans un énoncé sui-référentiel. Cela se fait dans des modalités différentes pour les deux types reconnus, mais a les mêmes effets quant à la négation:

«je ne dis pas/ne peux pas dire que je suis heureux de cela » [voire »...que je ne suis pas heureux », peu importe ici la polarité de la subordonnée],

«je n'exprime pas mon regret devant cette situation »

d'une part et d'autre part, «je ne te salue/remercie/félicite/... pas ». L'interprétation de ces énoncés (qui semblent surtout exprimer un refus de poser les actes en question) sera développée plus loin; constatons ici la simple possibilité de la négation pour les deux types, c'est-à-dire la neutralisation, pour les explicites, du contraste relevé au sein des actes primaires.

\subsubsection{Les actes représentatifs}

13 Leur but illocutoire est la description d'un état de fait. Cette caractérisation très floue y fait englober à nouveau des actes très hétérogènes.

14 A. Primaires : Les négations (polémiques ou descriptives) sont toutes naturelles, dans la mesure où les états de fait décrits se laissent plier à une représentation polarisée (c'est le problème de base de l'ontologie de la négation). Les exemples sont légion.

B. Explicites : je classe ici à nouveau les actes où l'énonciateur entre explicitement dans son énoncé en l'assumant. L'addition d'un marquage énonciatif de type " je déclare, je dis ... » etc. a des effets divers, qui font encore l'objet de discussions. En réalité, ce problème se pose déjà avec acuïté pour les actes expressifs explicites de type «je dis que je suis 
heureux ", où l'on aura rencontré les mêmes prédicats supérieurs. La classification des expressifs est sans doute à revoir (v. infra, 1.2.1.).

Ce qui compte ici, c'est que la négation de l'acte représentatif explicite a des effets remarquables: «ce mur n'est pas blanc» ou "vous n'êtes pas l'auteur de ce crime» (divers actes représentatifs primaires) ne sont point les équivalents de «je n'affirme pas que ce mur est/soit blanc » ou «je ne vous accuse pas de ce crime » (abstraction faite du potentiel performatif de ce dernier, mais qui ne me paraît pas être pour autant un acte déclaratif, même au positif). On voit que l'ancienne distinction entre négation interne et externe est à réinterpréter en termes pragmatiques (négation d'acte primaire ou explicite). Admettons provisoirement que l'interprétation semble à nouveau aller dans le sens d'un refus de poser les actes impliqués. Je ne tiens pas compte ici du phénomène de la «montée de la négation» affectant certains de ces énoncés complexes (R. Forest en traite ailleurs dans ce recueil).

\subsubsection{Les actes déclaratifs}

Ils regroupent les performatifs d'Austin, ayant pour but illocutoire de « rendre effectif le contenu de l'acte " ( $c f$ Moeschler 1985). Ils sont souvent institutionnalisés et leur aspect formulaire ne les fait pas apparaître, en règle, sous une forme primaire (de type «la séance est levée ») mais plutôt explicite («je lève la séance »). Qu'ils soient d'ailleurs primaires ou explicites, ces actes déclaratifs semblent en bloc très rétifs face à la négation, pour diverses raisons. Une raison pourrait être leur caractère figé, immuable, mais celui-ci indique encore leur rôle praxéologique, d'efficacité agissante. Si ces formules sont réservées pour rendre effectifs des actes (hautement conventionnels), le refus de les rendre effectifs exige sans doute des contextes et des expressions fort différents. Qu'on essaie de s'imaginer par exemple «Je ne vous déclare pas unis par les liens du mariage »!

On pourrait penser à une négation plus interne, qui viserait à rendre non effectif le contenu d'un acte, mais le même problème surgit: il s'agit d'actes institutionnalisés à caractère non réversible. Et puis il existe, si le cas se présente, une lexicalisation de l'acte approprié, avec le verbe « annuler ».

\subsubsection{Les actes commissifs}

Les commissifs ou promissifs ont pour but illocutoire «l'obligation contractée par le locuteur de réaliser une action future ».

A. Primaires : La négation des actes commissifs primaires ne pose à première vue aucun problème : «je ne viendrai pas » etc. Seulement, l'interprétation illocutoire (qui n'est déjà pas univoque pour «je viendrai ») est peu nette entre la négation externe ou interne (refus d'engagement ou promesse négative), voire entre d'autres lectures : avertissement, menace... L'addition d'une formule comme « sans faute » est difficile.

21 B. Explicites: "Je ne promets pas que je viendrai " se laisse lire comme le refus de contracter l'obligation. Il va de soi que la négation interne, en l'absence de «montée de la négation », donne un tout autre résultat («je promets que je ne viendrai pas »), qui ne nous concerne pas ici. 


\subsubsection{Les actes directifs}

22 Ils constituent le complément des actes promissifs, ayant pour but illocutoire de mettre l'interlocuteur dans l'obligation de réaliser une action future. Ce sont les seuls actes marqués par des modes verbaux ou des modalités phrastiques spécifiques. En réalité, en englobant tant les ordres que les questions, la classe est à nouveau très hétérogène. Le type d'obligation (communicative) qu'impose une question est différent de celui dans un ordre ; et leur comportement sous la négation est différent aussi.

23 A. Primaires : tant les actes interrogatifs que les injonctifs ont des rapports spécifiques avec la négation (décrits en détail dans Callebaut 1991, Ch. 1 et 2), mais il n'y a donc aucune incompatibilité de principe. Les ordres nous intéressent plus ici ; les questions ne sont en quelque sorte que les pendants modaux des assertions et leur caractère directif n'est qu'accessoire comparées aux ordres. Pour les impératifs négatifs, il y a des restrictions de type sémantique, mais qui ne vont pas en fait dans une seule direction: certains verbes favorisent l'ordre négatif et admettent mal (pour des raisons extralinguistiques) l'ordre positif : «?sois bête / ne sois pas bête ». Pour la discussion sur le caractère interne ou externe de la négation dans les injonctions, je suis Lyons (1977 : 773-775) qui y voit surtout des négations externes (la négation y visant à empêcher et à prévenir un acte, plutôt qu'à susciter un acte négatif : « Ne tombe pas !»).

B. Explicites : Possibles aussi bien pour les injonctifs que pour les interrogatifs, les actes directifs explicites expriment sous la négation le refus de mettre l'interlocuteur dans l'obligation en question: «je ne t'ordonne pas...", "Je ne demande pas...». Ceci indépendamment des lectures concrètes ( $\mathrm{p}$. ex. avec une négation polémique il peut $\mathrm{y}$ avoir une suite qui rectifie).

\subsection{Interprétation}

25 Il se laisse dégager deux grandes conclusions. La première concerne la typologie même des actes illocutoires, l'autre touche à la distinction entre actes primaires et explicites.

\subsection{1.}

On constate que les différents actes reconnus acceptent presque tous la négation (avec des effets variés, examinés plus loin). S'y soustraient pourtant en gros les actes expressifs primaires, du moins ceux que nous avons qualifiés de "performatifs", et les actes déclaratifs, qui ne sont autres que les performatifs originels d'Austin. La performativité est le fait d'actes exclusivement dirigés vers l'intervention active dans le monde; ils ont une valeur praxéologique très spécialisée, dont témoignent aussi les conditions d'accomplissement institutionnalisées et la forme figée qu'ils revêtent. On les voit donc mal accepter des négations pour accomplir des «non-actes" (au statut ontologique problématique). Seuls seraient envisageables des emplois négatifs pour annuler des actes réversibles, mais le langage préfère garder intacts les outils spécialisés de la performativité et trouve pour ces fonctions négatives d'autres expressions. Le clivage fondamental entre actes performatifs et constatifs trouve ainsi une justification dans leur comportement différencié face à la négation (mais il y aurait encore beaucoup à dire à ce propos). 
27 Accessoirement, on constate l'éclatement de la classe des actes expressifs (du moins pour leurs expressions primaires, mais on verra que pour les actes explicites, tous les types se confondent de toute façon). Il me semble possible de faire l'économie de la classe (souvent mise en doute déjà, il est vrai), en accueillant les actes expressifs constatifs parmi les actes représentatifs, et les actes expressifs performatifs parmi les actes déclaratifs. Ainsi, l'état émotionnel de l'énonciateur se range parmi les autres états de fait, et les actes de salutation, félicitation etc. rejoignent les performatifs classiques avec lesquels ils partagent la valeur praxéologique d'action sur le monde, l'aspect « ritualisé » et la forme figée. Mais que faire des interjections, qui sont, illocutoirement parlant, le noyau dur de la classe des « expressifs » (au sens jakobsonien déjà) ?

\subsection{2.}

La deuxième conclusion concerne donc l'intérêt de la distinction entre actes primaires et explicites. En effet, les distinctions entre types d'actes sont traversées par cet autre clivage. On a tout d'abord pu apprécier les différences de sens entre certains actes primaires niés et leurs "équivalents" explicites (cf "vous n'êtes pas l'auteur de ce crime » et « je ne vous accuse pas de ce crime»). Ensuite, les différences d'attitude envers la négation, relevées ci-dessus selon les types d'actes, ne s'affirment que pour les actes primaires. Les actes négatifs explicites, tous types confondus, ont un comportement remarquablement unifié. L'emploi de la négation y éclaire en plus la valeur propre de la démarche d'explicitation métalinguistique qui les caractérise.

Les actes explicites font intervenir l'énonciateur pour lui faire assumer l'acte en le nommant (sans doute en cas d'ambiguïté possible sur sa valeur) : en disant «je [Verbe illocutoire $\mathrm{X}$ ] que $\mathrm{Y}$ », je veux rendre clair que ma visée en communiquant ou en effectuant $\mathrm{Y}$ est de $\mathrm{X}$-er. La négation dans un acte primaire peut avoir divers effets, selon le type d'acte; dans l'acte explicite en revanche elle porte automatiquement sur la visée explicitée. La négation, conformément à son rôle habituel, focalise inévitablement sur l'intention illocutoire, et c'est pourquoi nous pouvons partout interpréter la négation illocutoire comme l'expression d'un refus. «Je ne promets pas que... » exprime le refus d'un engagement, «Je ne dis pas que... » le refus d'assumer un énoncé, «Je ne te félicite pas » le refus de porter une appréciation et d'entrer dans une certaine interaction etc.

Dans l'acte primaire, la négation a en quelque sorte un effet de type locutoire; elle n'affecte par exemple pas la valeur illocutoire d'ordre dans « N'avance pas ». Dans « je ne t'ordonne pas de faire cela ", étant ancrée dans un prédicat à fonction métalinguistique, elle affecte la valeur illocutoire de celui-ci. La négation me semble ainsi enlever toute valeur performative aux énoncés illocutoirement explicites qu'elle accompagne, ne leur laissant que la seule valeur du refus (mais au sens strict l'acte du refus n'est pas de ceux qui ont nécessairement et uniquement une expression langagière ; « je refuse... » n'est pas forcément un performatif). Dans ce sens, il est un peu malheureux que Lyons (1977 : 770) parle pour ces cas de "performative negation ", même si l'acte de "non-commitment " qu'elle aide à réaliser est lui-même un nouvel acte illocutoire. Cette négation détruit justement la performativité. Il apparaît ici encore que la négation est un méta-opérateur, intervenant a posteriori sur des énoncés qu'elle affecte de diverses façons. 


\section{Les prédicats à potentiel illocutoire et la négation}

\section{1 .}

Pour donner un peu plus de relief à la première partie, je présente ici quelques résultats d'un examen d'à peu près deux cents verbes. Je les ai recueillis en parcourant des listes de verbes de fréquence élevée en m'interrogeant sur leur potentiel illocutoire. Ils représentent grosso modo des équivalents français des SAV ("Speech Act Verbs») de Verschueren (1980 e.a.), ou les «Verbes illocutoires» de Roulet (1977), sans que j'aie repris strictement leurs différents critères, qui ne me convainquent pas entièrement. Je comprends par « verbes à potentiel illocutoire » :

a) tous les verbes susceptibles d'emplois performatifs (il s'agit toujours en cette matière d'emplois performatifs et non pas de verbes performatifs!) et

b) tous les verbes décrivant des actions essentiellement langagières, internes au locuteur (qui n'ont pas d'expression, de manifestation autres qu'à travers l'acte d'énonciation les nommant - même si leur réalisation n'en dépend pas forcément !).

L'examen a porté sur la réaction des énoncés (à la première personne et au présent) à l'égard de la négation, en rapport avec leur statut illocutoire (surtout sur le plan de la distinction performativité/ constativité). Un tel examen concret pose d'emblée beaucoup de problèmes : le jugement délicat de l'acceptabilité des énoncés testés, l'impossibilité de rendre compte des multiples facteurs dans une analyse forcément réductrice...

Le résultat primordial de cette analyse réside dans la reconnaissance d'un continuum allant de l'incompatibilité à l'acceptation, voire à l'affinité avec la négation. Il s'agit bien sûr toujours d'incompatibilité pragmatique (sauf peut-être pour certaines expressions figées qui n'offrent pas de point d'ancrage syntaxique à la négation, qui est en français assez exigeante sur ce point) et, puisque j'ai parlé de continuum, de tendances. La pragmatique reste le domaine des principes et non pas des lois. Ces tendances sont nuancées par un certain nombre de facteurs, point tous d'ordre illocutoire, même si pour les grandes lignes j'ai pu déceler un rapport avec des phénomènes illocutoires. Ces tendances ne valent d'ailleurs que pour les conditions illocutoires que j'ai définies au départ (énoncés à la première personne et au présent) : une négation " difficile » pour un performatif (ou, en général, à la première personne et au présent) peut ne poser aucun problème dans un emploi constatif (en changeant par exemple la personne), voire même à l'impératif (ce qui montre encore une fois la position très particulière des énoncés à la première personne) :

?Je ne décrète pas (de/que $\mathrm{X}$ )

Il n'a pas décrété de...

Je vous implore, ne décrétez pas de...

Ces exemples montrent déjà qu'il est un peu artificiel de créer des conditions de pure performativité pour les opposer à d'autres emplois. D'autre part, on voit déjà les nuances qui peuvent modifier ces conditions d'acceptabilité, p. ex. un ancrage temporel de type prospectif (avec un présent pour un futur) et une négation plus exclusive : « je ne décrète rien en cette matière avant le 1er janvier »... Comme la négation touche à beaucoup de phénomènes linguistiques, il n'y a pas que des facteurs illocutoires qui interviennent ici. 


\subsection{Conclusions}

2.2.1. souvent la performativité même de ces énoncés ; cela ne peut que favoriser l'acceptabilité de la négation. On en voudra pour preuve a contrario que les énoncés en question acceptent mieux des négations quand celles-ci sont visiblement de "seconde instance " (en réplique réfutative), et par excellence les négations «métalinguistiques ». Mais la négation métalinguistique est justement définissable comme celle qui porte sur l'énonciation et non pas sur l'énoncé; c'est-à-dire qu'elle détruira la performativité, performativité qui est le trait prépondérant de notre premier groupe.

?Je ne vous charge pas de faire $\mathrm{X}$.

vs

«- Ah, vous me chargez de faire $\mathrm{X}$ ?

- Je ne vous charge pas de cela, je vous en prie humblement. »

Ailleurs, la rareté des énoncés négatifs avec de tels verbes dérive du sens moralement répréhensible ou socialement réprouvé de ces négations : «je ne déplore pas ...« (si le complément est vraiment un fait déplorable), «je ne vous salue pas » etc. On quitte ici 
bien sûr le linguistique. Par ailleurs, ces restrictions extra-linguistiques opèrent aussi sur certains performatifs positifs (?"je vous insulte» etc.). Et à nouveau, la rhétorique exploite ceci dans l'autre sens (litote négative) pour atténuer des actes blamâbles ou menaçants (v. 2.2.3.).

\subsection{2.}

41 L'incompatibilité pragmatique avec la négation est donc, on l'aura vu, souvent relative. Pour toute une série d'autres verbes à potentiel illocutoire, cette difficulté ne surgit guère, et même dans les conditions précisées (première personne et au présent), la présence d'une négation semble aussi naturelle que son absence. Bien sûr, encore une fois, une négation polémique et a fortiori métalinguistique facilitent la chose, par leur ancrage dans un mouvement dialogique de réfutation. Mais la négation « de première instance ", la descriptive, apparaît aussi, pourvu simplement que l'état de choses présenté s'y prête selon son ontologie, sa polarisation. C'est le cas de verbes comme abandonner, accepter, admettre, accorder, accuser, s'adresser à, être d'accord, appeler ("nommer »), autoriser, blâmer, caractériser, choisir, citer,classer, commencer, compâtir, complimenter, comprendre, considérer, constater, se constituer, conseiller, contester, continuer, critiquer, croire, défendre, définir, demander, démentir, être en désaccord, désirer, deviner, dire, distinguer, écarter (une possibilité), écouter, embaucher, encourager etc. En fait, la démonstration devrait se faire pour tous ses verbes séparément, tant les facteurs intervenant sont multiples et d'appréciation délicate. On retrouvera notamment dans cette liste des verbes performatifs, comme annoncé dans le paragraphe précédent. Mais l'essentiel parait être constitué de verbes constatifs, et la taxinomie des deux classes me semble ainsi tirer une nouvelle justification de leur comportement différent à l'égard de la négation. Les énoncés constatifs sont bien plus ouverts à la négation que les performatifs. La facilité d'introduire la négation dans les énoncés constatifs n'appelle en fait pas de commentaires (à moins de revenir sur les questions fondamentales mêmes de l'ontologie négative et de la négation en langue). Et pour les performatifs de cette liste, une taxinomie plus fine, comme celle proposée par Dobrovie-Sorin (1985), s'avère pertinente. Elle distingue les " actes illocutionnaires conventionnels", les performatifs "archétypaux", des "non conventionnels ». Ce sont les premiers qui n'acceptent pas la négation; ceux de la liste qui l'acceptent mieux, autoriser, blâmer, complimenter, conseiller, critiquer, défendre/interdire, demander, embaucher, s'engager, insister, jurer (avec des réserves), licencier, observer, permettre, poser, promettre, rappeler etc., pour être performatifs, ne sont que peu institutionnalisés ou conventionnels. Il reste bien sûr, comme nous avons vu dans la première partie, que la négation qu'ils accueillent si bien, affecte toujours leur performativité même.

\subsection{3.}

42 J'avais annoncé que certains prédicats montrent une certaine affinité avec la négation. Il s'agit de verbes d'acte de parole qui jouent sur des combinaisons de négations lexicales et explicites pour des exploitations rhétoriques (d'atténuation), ou d'autres verbes où cette combinaison marche (dans des paires antonymiques) ou dont le sens se prête simplement bien à des polarisations négatives : apprendre (communiquer), assurer, garantir, cacher, cesser, contester, dire, insister, hésiter, manquer de, nier, s'opposer, pardonner, penser, permettre, se plaindre, plaider pour, prétendre, promettre, se prononcer, souhaiter, suggérer, trouver, vouloir. $\mathrm{Si}$ 
la place de certains verbes dans cette liste (toute provisoire) est moins évidente, c'est sans doute déjà que la corrélation avec la négation est plus sujette à caution. La statistique est encore à faire, je ne livre ici que des impressions. Ces tendances recoupent un peu les distinctions relevées; de toute façon, si de nouvelles conventions et un nouveau formalisme s'instaurent (en un sens rhétorique), la négation affecte toujours de la même façon la performativité.

\subsection{4.}

Une telle étude concrète, sortant des théories illocutoires, resitue la négation dans tout le champ de l'analyse sémantico-pragmatique. Ceci n'est qu'une étude-pilote, appelant de nombreuses nuances et vérifications. Mais, dans ce moment de relative désaffection pour les théories des actes de langage, elle va tout de même dans le sens d'une confirmation de l'intérêt des distinctions illocutoires (performatif/ constatif, primaire/explicite). Je ferais ainsi volontiers mienne la remarque d'I. Zagar (1991: 77), "You may find it surprising that 35 years after its discovery, I still consider performativity a source of wonder. » Mais cela ne vaut-il pas encore tellement plus pour la négation, " découverte » depuis plus de deux millénaires, et toujours source d'émerveillement?

\section{BIBLIOGRAPHIE}

BERRENDONNER A., 1981, « Zéro pour la question. Syntaxe et sémantique des interrogations directes ", CLF, $2: 41-67$.

BALLMER T.T., 1979, « Probleme der Klassifikation von Sprechakten » in GREWENDORF G., Sprechakttheorie und Semantik, Francfort : 264-273.

CALLEBAUT B., 1991, La négation en français contemporain. Une analyse pragmatique et discursive, Bruxelles, Palais des Académies [Publications de la Koninklijke Academie...,137].

DOBROVIE-SORIN C., 1985, Actes de langage et théorie de l'énonciation, Coll. ERA 642, Université de Paris 7.

LYONS J., 1977, Semantics, Cambridge, Cambridge University Press.

MOESCHLER J., 1985, Argumentation et conversation. Eléments pour une analyse pragmatique du discours, Paris, Hatier-Credif.

NICOLOFF F., 1984, Les performatifs explicites. Doctorat d'Etat (domaine : anglais contemporain), Université de Grenoble III.

RECANATI F., 1979, La transparence et l'énonciation. Pour introduire à la pragmatique, Paris, Seuil.

-, 1981, Les énoncés performatifs. Contributions à la pragmatique, Paris, Minuit.

ROULET E., 1977, « Des verbes illocutifs du français », Cahiers F. de Saussure, 31 : 247-257.

VERSCHUEREN J., 1980, On Speech Act Verbs, Amsterdam, J. Benjamins. 
ZAGAR I., 1991, "How to do Things with Words - The Polyphonic Way", in id. (Ed.), Speech Acts:

Fiction or Reality? (Proceedings of the Int. Conference, Ljubljana, 1990), IPrA:77-88.

\section{RÉSUMÉS}

Dans cette contribution, la théorie pragmatique illocutoire est examinée à la lumière du comportement des actes illocutoires face à la négation.

Il apparaît que les différentes interactions entre négation et type d'acte (vérifiées dans la typologie searlienne) montrent un continuum du rejet et de la négation jusqu'à l'affinité avec elle. Les résultats confirment l'intérêt de la distinction fondamentale entre actes performatifs et constatifs et entre actes explicités et primaires, et tendent à préciser certaines critiques des typologies illocutoires classiques.

L'étude plus concrète d'un certain nombre de verbes «à potentiel illocutoire " sous la portée d'une négation nous replonge dans les problématiques très diverses liées à la négation, et invite à aller plus loin dans l'étude des nombreux facteurs sémantiques et pragmatiques intervenant dans le comportement des énoncés négatifs.

This paper discusses the different interactions between negation and the illocutionary acts. It analyses the illocutionary values of negative utterances according to the classical taxonomy proposed by Searle. Some sentences show a rejection or, on the contrary, an affinity with negation, not indifferent to their illocutionnary status. The results confirm the interest of the initial distinction between performative and other acts, and between explicit and primary ones and so sheds some light on the functioning of negation in general.

The second part focusses attention on "speech act verbs" under negation, and hints to the diversity of semantic and pragmatic aspects involved.

\section{AUTEUR}

\section{BRUNO CALLEBAUT}

Université de Valenciennes 